\title{
Comparison of Absolute Distance Measurement by Different Types of Dual Mode-Locked Fiber Lasers
}

\author{
Tze-An Liu, Yi-Chen Chuang, Hau-Wei Lee, Po-Er Hsu and Jin-Long Peng \\ Center for Measurement Standards, Industrial Technology Research Institute, Hsinchu 300, Taiwan
}

\begin{abstract}
Two types of the dual mode-locked fiber lasers for asynchronous absolute distance measurement are investigated. The lasers are linear and ring cavity with repetition rate of $70 \mathrm{MHz}$ and $100 \mathrm{MHz}$, respectively. The group velocity dispersion is not compensated in the first type of the lasers, while the others are fully done. The timing jitter with the Allan deviation below averaging time of $0.2 \mathrm{~s}$ during the distance measurement for around $1 \mathrm{~m}$ of both types of lasers were $2.5 \mathrm{ps}$ with $600 \mathrm{~nm}$ and $1.6 \mathrm{ps}$ with $200 \mathrm{~nm}$. We concluded that the phase noise resulted from the intra-cavity dispersion is the main contribution for the uncertainty of the ranging in these two types of the lasers.
\end{abstract}

Key words: Mode-locked, fiber laser, distance, timing jitter.

\section{Introduction}

Precise absolute distance measurement is very crucial for large-scale manufacturing. Classic multi-wavelength interferometry can be slow and subject to cyclic errors. In 2009, Coddington et al. [1] had demonstrated the precise ranging with precision of $\sim 200 \mathrm{~nm}$ by utilizing dual fully frequency stabilized $100 \mathrm{MHz}$ fiber laser comb. Dual-comb based techniques, a subclass of the cross-correlation techniques, are particularly attractive because they can scan an entire range window quickly allowing for rapid update rates against multiple targets, and requiring no balancing of interferometer paths [2, 3]. Later, a simple linear cavity without any GVD (group velocity dispersion) compensation, dual $200 \mathrm{MHz}$ free running mode-locked fiber lasers are demonstrated [4]. The dual lasers are utilized for the ranging and the precision is also $\sim 200 \mathrm{~nm}$ in averaging time of $\sim 20 \mathrm{~ms}$. Recently, a dual-comb nonlinear asynchronous optical sampling technique was demonstrated for the absolute distance measurement [5]. The pulse intensity was acquired directly from the time of flight measurement, and the

Corresponding author: Tze-An Liu, Ph.D., senior researcher, research field: dual mode-locked fiber laser based absolute distance measurement. data processing time was reduced without using Hilbert transform. However, the high power laser is required for the nonlinear frequency doubling effect. The repetition rate is stabilized to $250 \mathrm{MHz}$. It was also used for the ranging, and the precision was similar as the former cases. These three papers were with long pulse repetition rate, the ambiguity was smaller than 1 $\mathrm{m}$.

In this paper, we utilized longer linear laser cavity with lower repetition rate of $70 \mathrm{MHz}$ to obtain the larger non-ambiguity range of $2.1 \mathrm{~m}$ by asynchronous sampling technique. It is housing in a compact box for the long range industrial applications. The results are compared with a well GVD compensated ring cavity with a similar repetition rate of $100 \mathrm{MHz}$.

\section{Experimental Apparatus}

\subsection{Laser Design}

We use two types of the lasers for the absolute distance measurement. All of the experimental setups are described in the following. The first type of the lasers is designed as a linear cavity mode-locked laser without any dispersion compensation. The experimental set up is depicted in Fig. 1. The laser employed here is an Erbium all fiber design operating at 
repetition rates of $\sim 70 \mathrm{MHz}$, which corresponds to the time of flight non-ambiguity range of $2.1 \mathrm{~m}$. The cavity design is a semiconductor SAM (saturable absorber mirror) based linear-cavity modeled loosely on Ref. [6] (see Fig. 1). Each end facet of the fiber cavity is connectorized with a pc connector. One end of the laser cavity is formed by sandwiching a SAM between the pc connector at the fiber end and a second pc connector, which is known as the butt-coupled technique. The output coupler at the other end of the laser cavity is similarly formed by another pc connector, which is butt-coupled with a cover glass plate coated with 35 nanometers of gold. The gold coated facet is directly connected to the pc connector of the cavity end. It is sufficient to monitor the repetition rate of only a single laser for the self-calibrating data analysis technique in the ranging applications. Therefore, we designed two output ports. One output port is the gold reflector (Out 1 ), and the other is the WDM (wavelength division multiplexing) port of the PBS (polarizing beam splitter) rejection channel (Out 2). An intra-cavity polarizer simultaneously provides the polarization selectivity and an input channel for the $1,480 \mathrm{~nm}$ pump light. The gain is provided by a $31 \mathrm{~cm}$ section of highly doped (80 $\mathrm{dB} / \mathrm{m})$ Er fiber with $8 \mu \mathrm{m}$ core diameter. One of the lasers employed a tunable optical delay for the cavity length adjustment, and resulted in the repetition rate difference tunable from $0 \mathrm{~Hz}$ to $500 \mathrm{kHz}$ for the asynchronous sampling.

The difference in repetition rate of both lasers is tuned to $\sim 1 \mathrm{kHz}$, providing an experimental update rate of only $\sim 1 \mathrm{~ms}$. Repetition rates of both lasers are not stabilized by any controller and just roughly measured by frequency counters. The lasers were housed in a box as shown in Fig. 2 with dimension of $32 \times 28 \times 9.5 \mathrm{~cm}^{3}$ to protect them from air currents and robust handling, but no temperature control or active feedback was used to otherwise stabilize their output.

The laser is started lasing with pumping LD (laser diode) current $\sim 100 \mathrm{~mA}$. As the pumping current tuned up to $200 \mathrm{~mA}$, the fiber laser is started mode-locking.

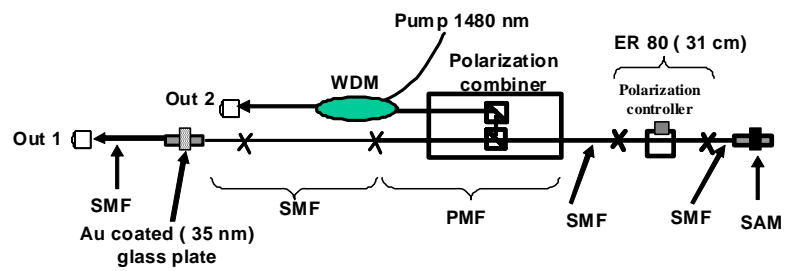

Fig. 1 Design for the fiber laser. SMF-single mode fiber, PMF-polarization maintaining fiber, SAM-saturable absorber mirror.

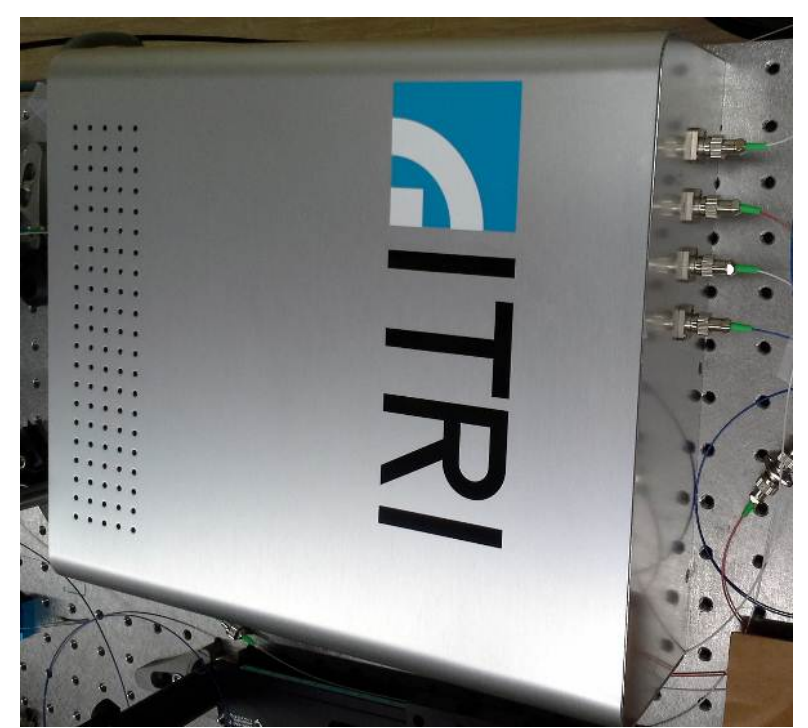

Fig. 2 Real picture of the housing for the dual mode-locked fiber lasers.

After fine tuning the polarization controller, the output power is increased up to $12 \mathrm{~mW}$ as the pumping current increased to $370 \mathrm{~mA}$. The stable RF (radio frequency) spectrum with a fundamental frequency of $\sim 70 \mathrm{MHz}$ of the laser is acquired from the detector that impinged by the output of gold reflector. It can be employed as the monitor port of the laser repetition rate via $\mathrm{RF}$ frequency counter by further filtering and amplifier during the absolute distance measurement applications.

The second type of the lasers is designed as a ring cavity mode-locked laser with full dispersion compensation. The dual laser cavities are designed as similar as our previous laser [7]. The dispersion is well controlled to around zero with comparison to the previous design of positive GVD. An SAM for sustaining the mode-locking easier was also utilized in this cavity. The output power of dual lasers is $>10 \mathrm{~mW}$ with pulse width $<100$ fs. 


\subsection{Absolute Distance Measurement}

The absolute distance measurement is realized by using these dual ultrafast fiber lasers in a time-domain down-sampling configuration [1] as shown in Fig. 3. The probe laser is retro-reflected off a pc connector and a movable corner cube to form the measurement path. The overlap between the two reflected probe pulses and LO (local oscillator) pulses is digitized synchronously with the LO comb pulses and stored for analysis. The output signal was connected to an LPF (low pass filter) to meet the Nyquist sampling condition [1]. The beating signal to noise ratio is $\sim 20 \mathrm{~dB}$, and is ready for the data acquisition.

The analyzing procedure is shown in Fig. 4. The raw data are Hilbert transformed to generate the imaginary component of the complex analytic representation of the signal from the real part. The carrier oscillations can then be removed by taking the modulus of this complex analytical signal to leave only the modulus, or signal envelope. The different pulses are then fit with a series of Gaussians to find the peak centers across the interferogram. For these well-behaved spectra, a Gaussian fit was well matched to the observed shape. We identify a target and reference reflection and, for each interferogram, calculate the time delay between the target and reference pulse (pk11) and the time delay between subsequent reference pulses (pk12) both in units of the pulse sample number. The distance is calculated as shown in Fig. 4.

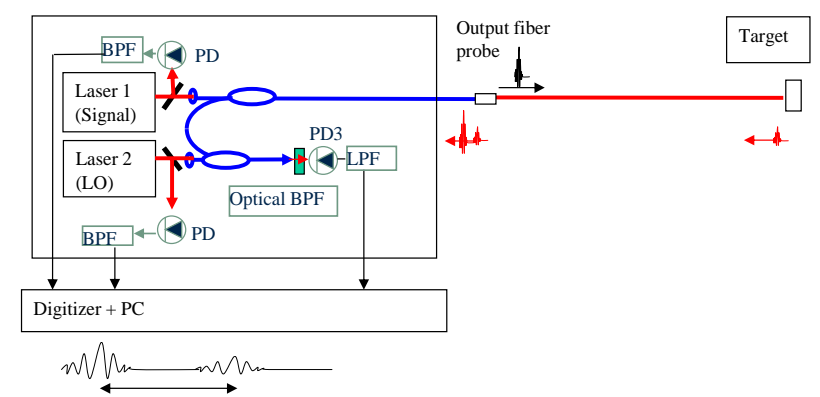

Fig. 3 Schematic diagram of the setup for absolute distance measurement.

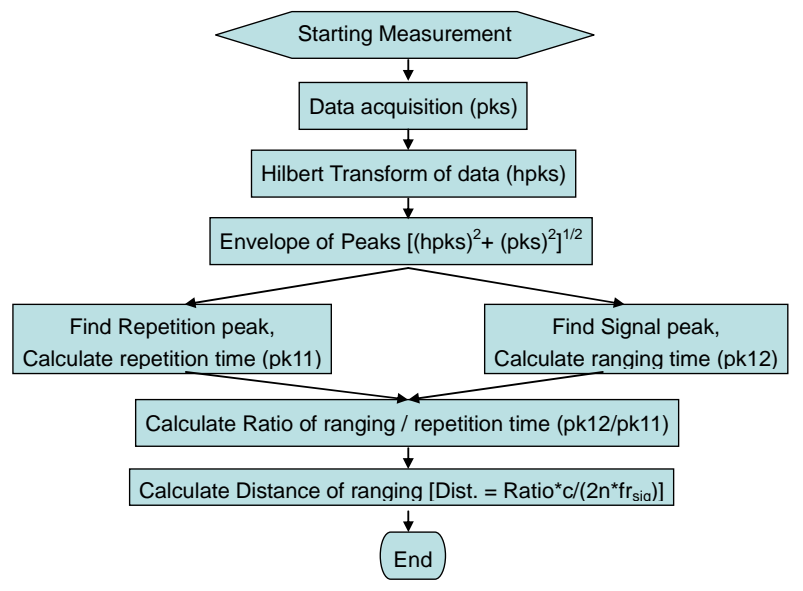

Fig. 4 Flow chart for the data analysis.

\section{Experimental Results and Discussions}

3.1 Ranging Uncertainty and Timing Jitter of the Non-dispersion Compensated Linear Cavity Laser

The envelope of peaks for measuring the absolute distance is $\sim 1 \mathrm{~m}$. The Allan deviation is calculated from the data as shown in Fig. 5.

The measurement precision is $20 \mu \mathrm{m}$ at the minimum acquisition time of $0.8 \mathrm{~ms}$ (set by the $1.25 \mathrm{kHz}$ difference in laser repetition rates), and dropping $\sim 600 \mathrm{~nm}$ at $200 \mathrm{~ms}$ averaging periods. The result is not as good as our previous ranging data by higher repetition rate with shorter fiber length design [4]. It might be because of the much more positive dispersion induced phase noise in the longer fiber length of $70 \mathrm{MHz}$ laser. The uncertainty of the distance

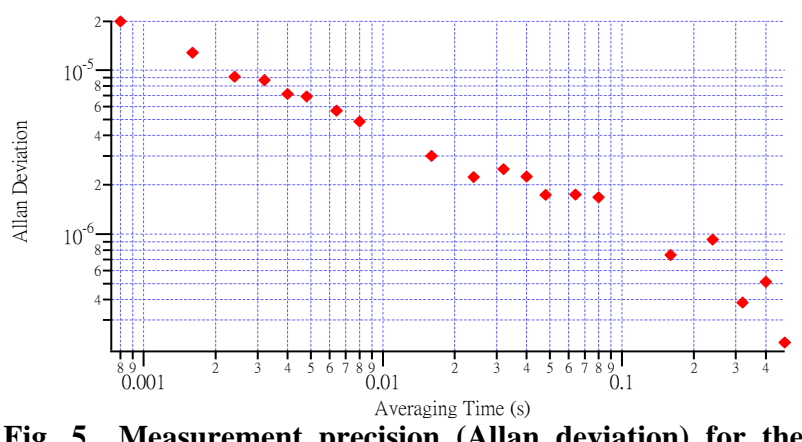

Fig. 5 Measurement precision (Allan deviation) for the distance of $\sim \mathbf{1} \mathrm{m}$ by dual non-dispersion compensated mode-locked fiber lasers. 
measurement by pulse time of flight method is mainly from the timing jitter. The timing jitter, which calculated from the phase noise, is attributed by many reasons [8]. The relative crucial parameter is the cavity GVD.

We employed a commercial phase noise measurement machine (PN9000) for checking the timing jitter of the laser. The phase noise with respect to the frequency is shown in Fig. 6. The vertical axis is the phase noise PSD (power spectral density, $\mathrm{dBc} / \mathrm{Hz}$ ), and the horizontal axis means the noise frequency. The signal is acquired from the repetition rate of the linear cavity without dispersion compensated mode-locked fiber laser. The integrated PSD (i.e., timing jitter) from $1 \mathrm{~Hz}$ to $1 \mathrm{MHz}$ is about $2.5 \mathrm{ps}$.

\subsection{Ranging Uncertainty and Timing Jitter of the Well} Dispersion Compensated Fiber Laser

We compare the ranging uncertainty with the ring cavity type, dual zero dispersion $100 \mathrm{MHz}$ free

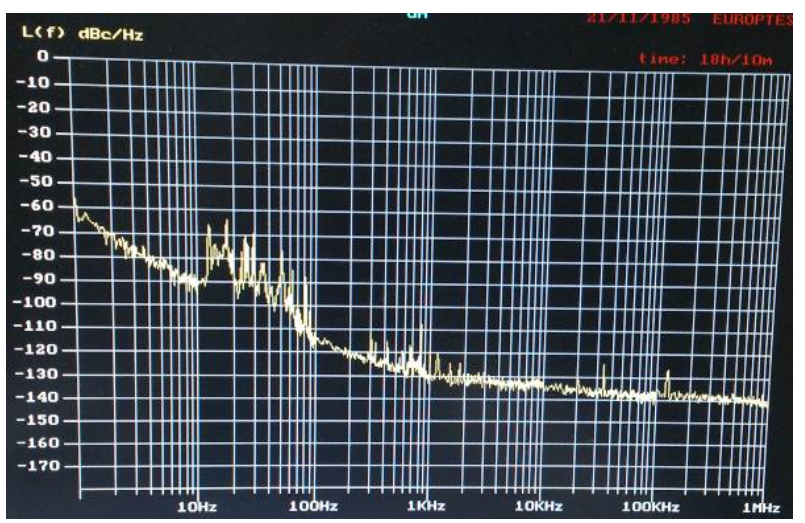

Fig. 6 Phase noise of the $70 \mathrm{MHz}$ non-dispersion compensated mode-locked fiber laser.

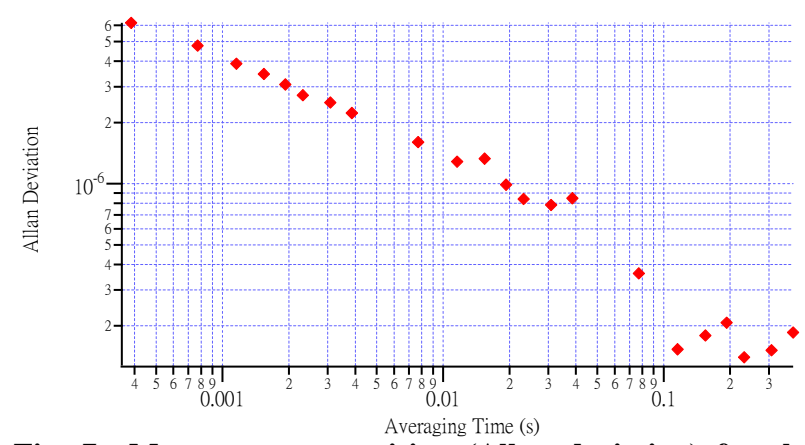

Fig. 7 Measurement precision (Allan deviation) for the distance of $\sim \mathbf{1} \mathbf{m}$ by dual zero GVD mode-locked fiber lasers. running mode-locked lasers. The calculated Allan deviation from the ranging of $\sim 1 \mathrm{~m}$ data is shown in Fig. 7. The measurement precision is $6 \mu \mathrm{m}$ at the minimum acquisition time of $0.4 \mathrm{~ms}$ (set by the 2.5 $\mathrm{kHz}$ difference in laser repetition rates), and dropping $200 \mathrm{~nm}$ at $200 \mathrm{~ms}$ averaging periods. It is better than our non-dispersion compensated laser performed distance measurement.

The repetition rate of the ring cavity with zero GVD mode-locked fiber laser was acquired from a photodetector. It is connected to a commercial phase noise measurement machine for checking the timing jitter of the laser. The phase noise with respect to the frequency is shown in Fig. 8. The vertical axis is the phase noise PSD $(\mathrm{dBc} / \mathrm{Hz})$, and the horizontal axis means the noise frequency. The signal is acquired from the repetition rate signal of the ring cavity with well dispersion compensated mode-locked fiber laser. The integrated PSD (i.e., timing jitter) from $1 \mathrm{~Hz}$ to 1 MHz is about 1.5 ps.

The timing jitters of these two types of the mode-locked lasers are 2.5 ps and 1.5 ps, respectively. The larger timing jitter in the $70 \mathrm{MHz}$ laser is mostly attributed to the larger phase noise during the frequency of $10 \mathrm{~Hz}$ to $100 \mathrm{~Hz}$. It is also possibly resulted from the environmental low frequency vibration noise. However, it is acquired in the same laboratory and nearly at the same time, therefore, it should be mostly resulted from the GVD induced noise. Shi et. al. [9] also demonstrated that the measured uncertainty by

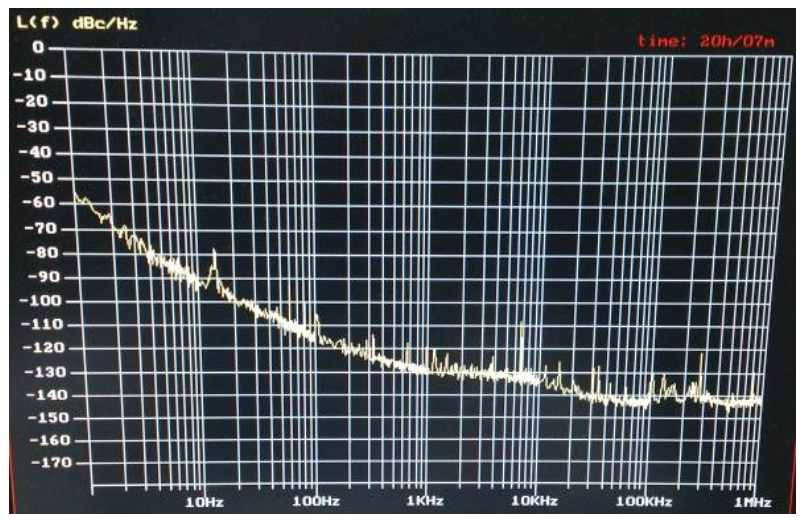

Fig. 8 Phase noise of the 100 MHz zero GVD mode-locked fiber laser. 
dual comb technique is significantly affected by different type of the dispersion. Both types of lasers are not temperature controlled. Although the timing jitter between these types of the lasers is only about 1.6 times, the uncertainty of the time of flight ranging is about 3 times. It could be explained that the asynchronous sampling requires dual laser for the ranging, the timing jitter induced time of flight uncertainty will thus be doubled. Although our previously $200 \mathrm{MHz}$ laser performed better results [4], the cavity length is shorter than our new $70 \mathrm{MHz}$ laser, and performed much smaller dispersion and uncertainty during the ranging.

\section{Conclusions}

We had established dual compact and repetition rate difference tunable free running mode-locked fiber laser based ranging system. The pulse time-of-flight yields a measurement precision of $600 \mathrm{~nm}$ with non-ambiguity range of $2.1 \mathrm{~m}$ in $200 \mathrm{~ms}$ for distance of $\sim 1 \mathrm{~m}$. The calculated Allan deviation below averaging time of 0.2 $\mathrm{s}$ during the distance measurement of $\sim 1 \mathrm{~m}$ with the timing jitter compared to $100 \mathrm{MHz}$ ring cavity with zero GVD mode-locked fiber lasers were $600 \mathrm{~nm}$ with 2.5 ps and $200 \mathrm{~nm}$ with $1.6 \mathrm{ps}$, respectively. We concluded that the uncertainty of the ranging is attributed to the intra-cavity dispersion induced phase noise.

\section{Acknowledgment}

This research was supported by the Bureau of
Standards, Metrology and Inspection of the Republic of China (Taiwan).

\section{References}

[1] Coddington, I., Swann, W. C., Nenadovic, L., and Newbury, N. R. 2009. "Rapid and Precise Absolute Distance Measurements at Long Range.” Nature Photonics 3: 351-6.

[2] Yokoyama, S., Yokoyama, T., Hagihara, Y., Araki, T., and Yasui, T. 2009. "A Distance Meter Using a Terahertz Intermode Beat in an Optical Frequency Comb.” Optics Express 17: 17324-37.

[3] Godbout, M., Deschenes, J. D., and Genest, J. 2010. "Spectrally Resolved Laser Ranging with Frequency Combs.” Optics Express 18: 15981-9.

[4] Liu, T.-A., Newbury, N. R., and Coddington, I. 2011. "Sub-micron Absolute Distance Measurements in Sub-millisecond Times with Dual Free-Running Femtosecond Er Fiber-Lasers.” Optics Express 19: 18501-9.

[5] Zhang, H., Wei, H., Wu, X., Yang, H., and Li, Y. 2014. “Absolute Distance Measurement by Dual-Comb Nonlinear Asynchronous Optical Sampling.” Optics Express 22: 6597-604.

[6] Hartl, I., Imeshev, G., Fermann, M. E., Langrock, C., and Fejer, M. M. 2005. "Integrated Self-referenced Frequency-Comb Laser Based on a Combination of Fiber and Waveguide Technology.” Optics Express 13: 6490-6.

[7] Peng, J.-L., Ahn, H., Shu, R.-H., Chui, H.-C., and Nicholson, J. W. 2007. "Highly Stable, Frequency-Controlled Mode-Locked Erbium Fiber Laser Comb.” Applied Physics B 86: 49-53.

[8] Paschotta, R. 2010. "Timing Jitter and Phase Noise of Mode-Locked Fiber Lasers.” Optics Express 18: 5041-54.

[9] Shi, H., Song, Y., Liang, F., Xu, L., Hu, M., and Wang, C. 2015. "Effect of Timing Jitter on Time-of-Flight Distance Measurements Using Dual Femtosecond Lasers.” Optics Express 23: 14057-69. 